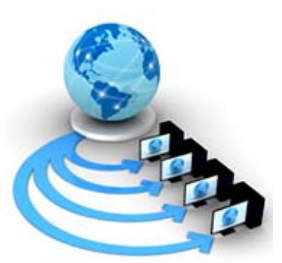

Volume 8, No. 8, September-October 2017

International Journal of Advanced Research in Computer Science

RESEARCH PAPER

Available Online at www.ijarcs.info

\title{
A SURVEY ON NEW APPROACHES OF INTERNET OF THINGS DATA MINING
}

\author{
Sallauddin Mohmmad \\ Assistant Professor, \\ Department of Computer Science \& Engineering , \\ SR Engineering College, \\ Warangal,Telangana,India
}

\author{
G. Sunil \\ Assistant Professor, \\ Department of Computer Science \& Engineering , \\ SR Engineering College, \\ Warangal,Telangana,India
}

\author{
Ranganath Kanakam \\ Assistant Professor, \\ Department of Computer Science \& Engineering, \\ Sumathi Reddy Institute of Technology for Women, \\ Warangal,Telangana,India
}

\begin{abstract}
The huge information created by the Internet of Things (IoT) is taken for high business appraisals, and data mining calculations can be related to IoT to separate hidden data from data. IoT data mining in learning view , method view and application view along with grouping, bunching, time management and exception analysis. Many number of devices are associated with IoT, massive amount of volume data producing every day.Data Mining is a process of mining required data from databases in efficient way and obtaining optimized results. In subsequently Internet of Things (IoT) Middleware applications allow data user to retrieve and read the data without even knowing about devices ,signaling ,communication and technical issues in IoT resources data processing . Configuring an IoT middleware layer and retrieving data is a big challenge for data consumers .In this paper, we survey on how IoT data can be produce and in such away automated knowledge discovery from data storage .
\end{abstract}

Keywords: IoT, Data mining, Data Process , IoT Middleware applications.

\section{INTRODUCTION}

The Internet of Things (IoT) [2] collection of connecting computing devices. The devices in IoT are basically with sense and actuate. Now a days there are about 1.5 billion Internet-enabled PCs, more than 1 billion mobile phones and many sensor devices equipped to different architectures ITS, Smart Grids, SWM, environmental monitoring ,agriculture, and manufacturing etc . we can predict that by 2020 IoT creates 50 to 100 billion devices connected to the Internet [2].In order to understand, analyze, store, retrieve and process the data generated from IoT devices needs to beefed into more appropriate data analytic applications. Such applications are designed to process and produce certain results once they are given to sensor data as inputs. Many IoT middleware solutions using for retrieval of data from sensors in a simplify manner and they are acting as a mediator between the hardware of IoT devices and the application layer. these middleware applications need to be configured depends on the context of sensor information and user requirements.

IoT devices shared with Cloud Computing for utilize the services, features of Cloud such as provisioning ,scalability , elasticity etc. Cloud provisioning feature provides a model of IoT t IoT cloud creates sensing as a service and creates multiple types of sensor they can sense, fusing, filtering, and reasoning mechanisms together by on-demand. To utilize the cloud services and features we need of appropriate IoT middleware. That should be in users perspective can collect sensor data streams ofter next inject data into data repositories . when user require it should be extract with suitable analytical process[4]. In real time the data stream is a set of data segments that are captured and produced sequentially and continuously at certain time intervals .time intervals may be like every 5 seconds, every few hours.

One of the best job of an IoT middleware is to combine all sensors and data processing components and subsequently produce a data stream. Mostly data streams that consists same data elements but it consists multiple data types. Sensor data feed the data stream into an appropriated application for further data processing such as modeling and visualization that allows the data users to achieve their objectives.

Data encoding (O\&M and JSON). Data will encoded and directly be used GIS also served in SOS as O\&M. Already available XML encodings for the O\&M and JSON data model[21].

Sensor metadata (SensorML). SensorML model allows encoding then provisioning of metadata about sensors.the measurement procedure in a XML format[21]. 


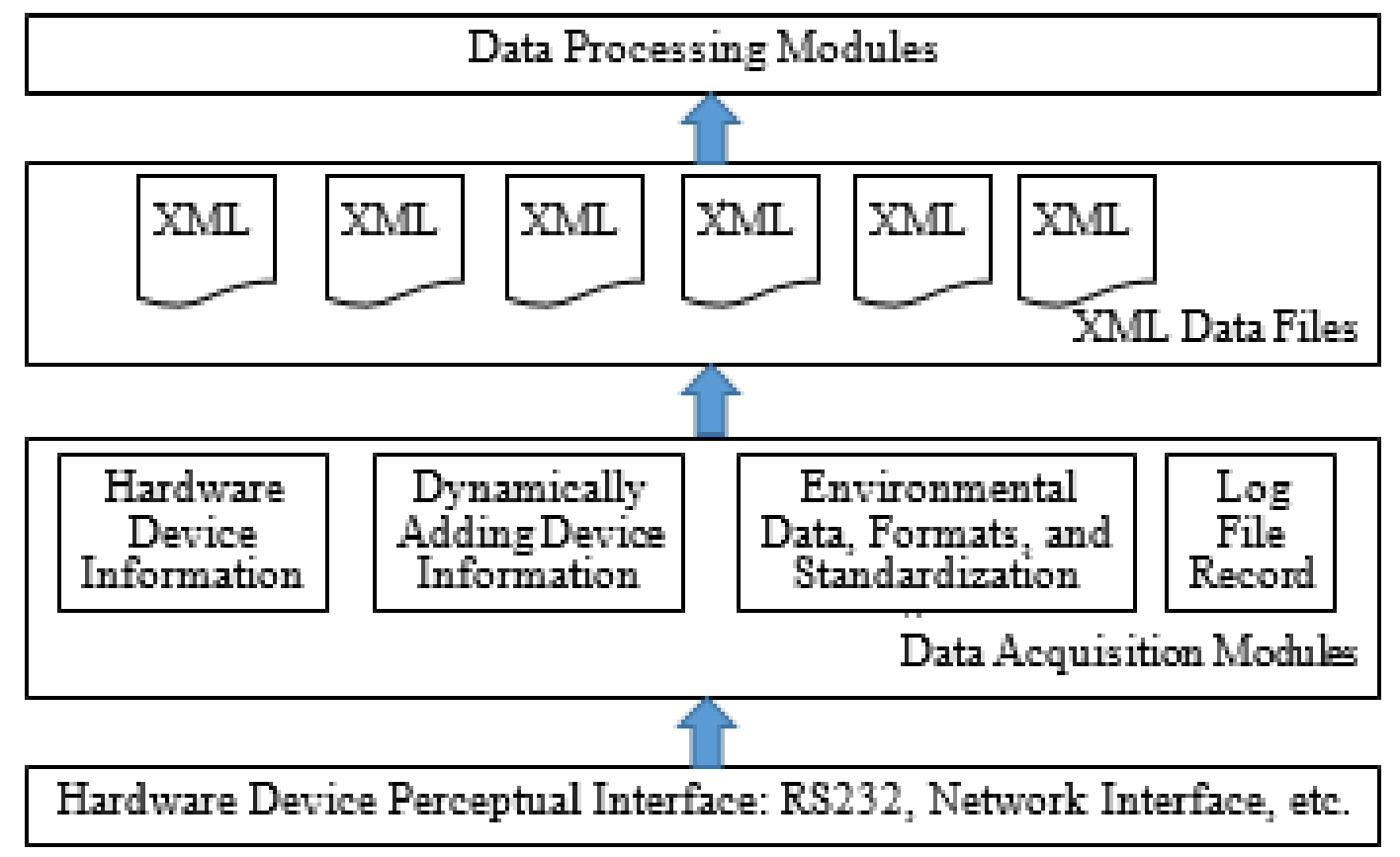

Figure 1. IoT data at user interface .

From IoT is a collection of sensor networks and devices included with RFID[25]. The RFID data added with EPC, location, time. location represents the place where the reader is positioned. EPC helps for dentifying RFID uniquely by an RFID reader .and time used to represent the exact time interval when the reading took place.

18 bytes of memory required to store the raw RFID record. So for a RFID data stream of a ITS, IWM , supermarket etc .. [24]will produce the Peta Bytes of data for every second, then we can imagine that how much of data producing per a day . So that it is necessary to develop effective methods for analyzing, managing and mining RFID data. The data of Internet of Things can be expressed into several types: address/unique identifiers , RFID data stream, positional data, descriptive data, sensor network data and environment data etc[26][27] . It creates great challenges for analyzing, managing and mining data in the Internet of Things.

The Internet of Things (IoT) and its related technologies integrated with public networks. These days it also has attracted the attention of researchers from academics, industries and government in recent years. There is a huge perception that things are controlled and monitored easily and are identified clearly[4]. They are capable of communicating with each other through internet, and can even make own decisions. In order to expand and improve IoT, lots of technologies are introduced, among them data mining is one of the important technique. Data mining involves finding the info, like novels, and useful patterns and applying few algorithms in order to fetch the required data.

The main functionality of data mining process is that build an appropriate predictive or descriptive model for massive data that not only satisfies the user needed data but also can be able to generalize the new data[5]. data mining is a process of retrieving the usable patterns of knowledge from huge amount of data stored in data warehouses [8]. On merging of all data mining functionalies defines data mining process by following steps .
1. Data preparation: Preparing the data is basic process which includes 3 sub steps:

- Integrate collected data from various inputs and for accuracy clean the noise from data .

- Extract some parts of data into data mining system.

- $\quad$ Preprocess the data for data mining.

2. Data mining: Apply appropriate algorithms to the information which is available at repositories to be mined to discover the Knowledge .

3. Data introduction: envision the data and speak to mined learning to the consumer. we will see info mining in an exceedingly multi dimensional view. In application, it incorporates business, media transmission, managing an account, deceit examination, biodata mining, stock exchange investigation, content mining, web mining, social organization, and net business.

The assortment of researchers concentrating on learning view, procedure view and application view can be found in the writing. Nonetheless, no past effort has been created to audit the distinctive views of data mining expeditiously, particularly in recently large data. Portable web and internet of Things develop quickly and a few data mining scientists move their thought from data mining to large data. There are many data cube measure to load of data which will be store and mined which may be like social database, multimedia databases, No SQL database[23], information distribution center, content and web interactive media ,heritage framework log ,world Wide web and it is also frominternet of Things information,. Galvanized by this during this paper we tend to endeavor to create a whole study of the crucial late advancements of knowledge mining examine. This summary concentrates on information used strategies view and application perspective of data mining[8]. The purpose of this paper incorporates three sections: the primary half is that we tend to propose a unique approach to survey info mining in info see method view and application; the second half is that we tend to quote the new attributes of big info and break down the difficulties. Another essential 
commitment is that we tend to propose a suggested large info mining framework. it's necessary for per users on the off probability that they have to create a enormous data mining framework with open source advancements.

IoT and large data square measure talked concerning totally, the new advances to mine enormous data for IoT square measure reviewed, the difficulties in vast data time square measure outlined, and another monumental data mining framework design for IoT is proposed.

\section{DATA MINING MODELS FOR THE INTERNET OF THINGS}

IoT Multi-layer model for data mining according to the architecture of IoT and data mining framework of RFID[24] . Four layers are data collection layer, data management layer, event processing layer and data mining service layer. data collection layer contains devices, e.g. RFID Reader and sinks. This layer collect various smart object's data, which are GPS data, RFID stream data, satellite data, sensor data and positional data[26] . Data management layer used for centralized or distributed database or data warehouse to manage collected data. After the object identification, compression and data abstraction, various data are saved in the corresponding data warehouse. Take RFID data as an example, the raw format of RFID data stream is (EPC, location, time), where EPC marks smart object's ID. After data cleaning, we can obtain Stay table which contains records as the format (EPC, location, time in, timeout). Here $\mathrm{XML}$ language can be used for presenting data in IoT.In IoT devices are communicated via the data management layer to claim and store the data[21].

Event is combination of combines data, time etc. So it should process in high-level mechanism for data of IoT. Event processing layer defined for event process and analyze events effectively. In that way we are performing event-based query or analysis in that layer. Data mining service layer is constructed based on event processing and data management. Various event-based mining procedures and services, such as clustering, association , classification, forecasting and outlier detection are accommodated for applications.

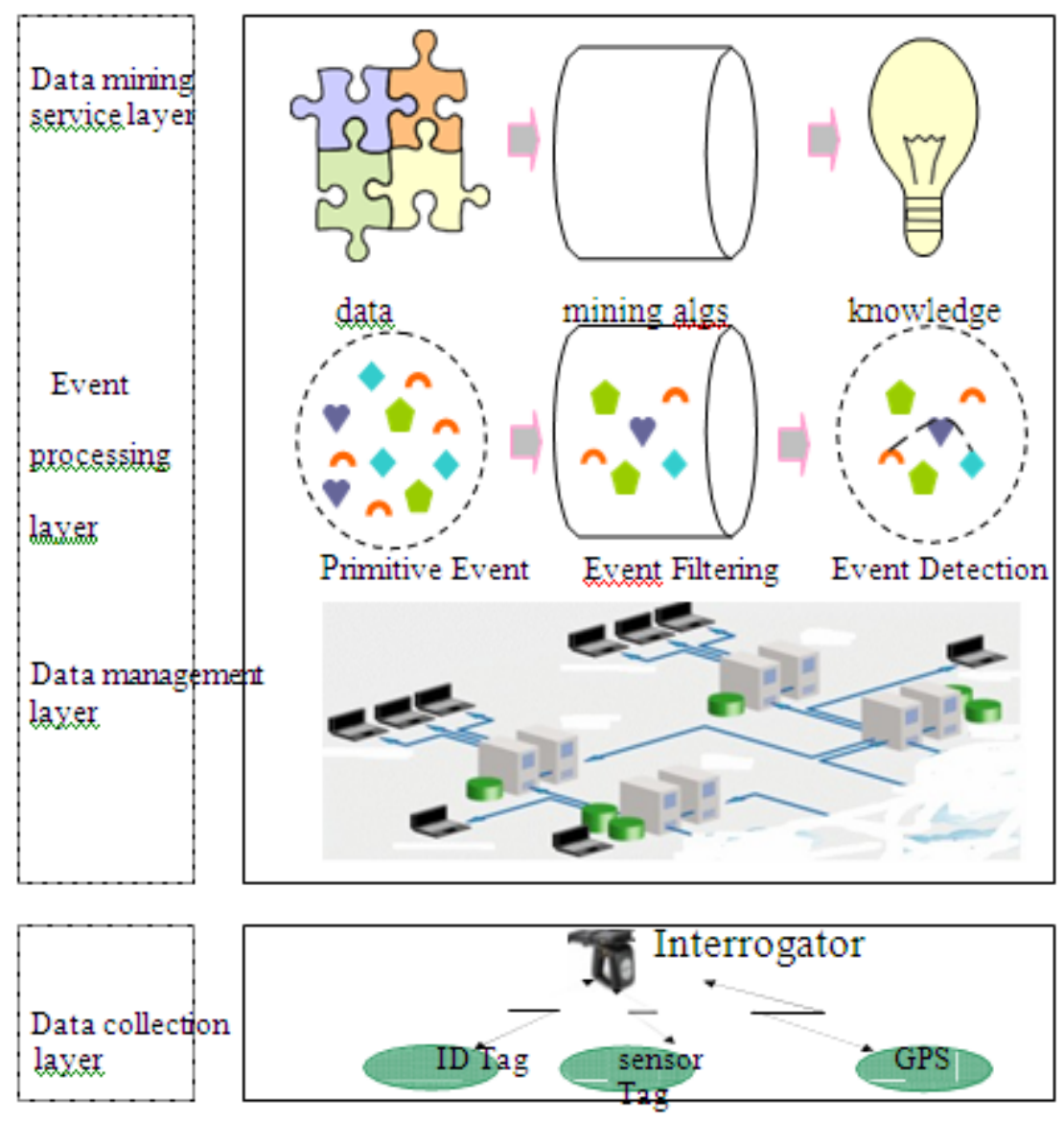

Figure 2. Data Mining model for IoT

2.1. Classification: Order is important for administration of basic leadership. Given a question appointing it to at least one of predefined target classifications or classes is named classification[18] .The goal of order is to precisely foresee the objective class for every case within the information . for instance a arrangement model can be utilized to recognize credit candidates as low, medium, or high credit dangers .
There are numerous methods to cluster the data as well as choice tree time period, outline based mostly\} or administers based master frameworks numerous leveled characterization neural systems, Bayesian system, and bolster vector machines 


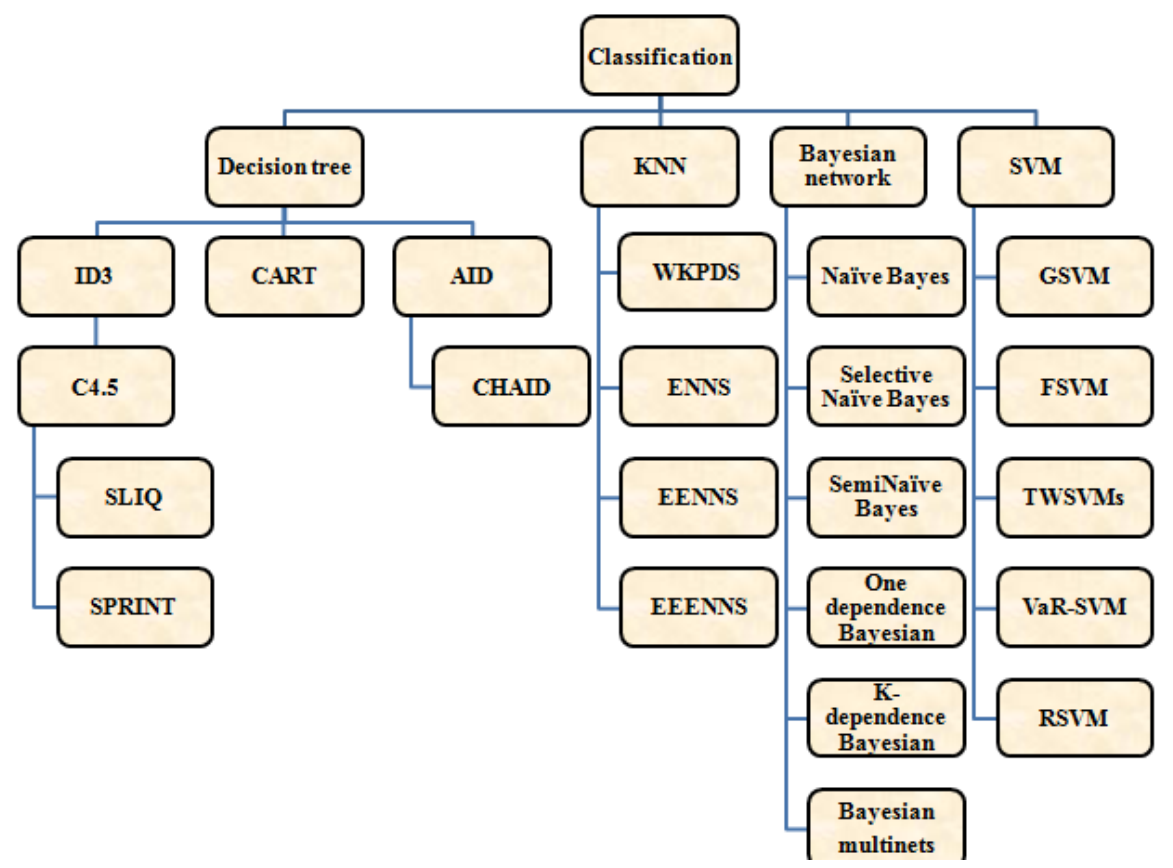

Figure 3. Research structure of Classification

- $\quad$ CHAID (chi-squared programmed collaboration finder) and therefore the change scientist concentrate on separating associate degree informational assortment into elite and thorough sections that contrast regarding the reaction variable.

- In view of Bayesian systems these classifiers have various qualities almost like model interpretability and convenience to advanced data and order issue settings[13]. The examination incorporates Thomas Bayes, particular Bayes, one-reliance Bayesian classifiers , K-reliance Bayesian classifiers, Bayesian organize increased Bayes, unlimited Bayesian classifiers and Bayesian multi nets.

Support Vector Machines calculation is run learning model with connected learning calculations that dissect data and perceive designs that is in light of measurable learning hypothesis[14]. SVM produces a parallel classifier the alleged ideal analytic hyper planes through associate astonishingly nonlinear mapping of the knowledge vectors into the high-dimensional highlight house.

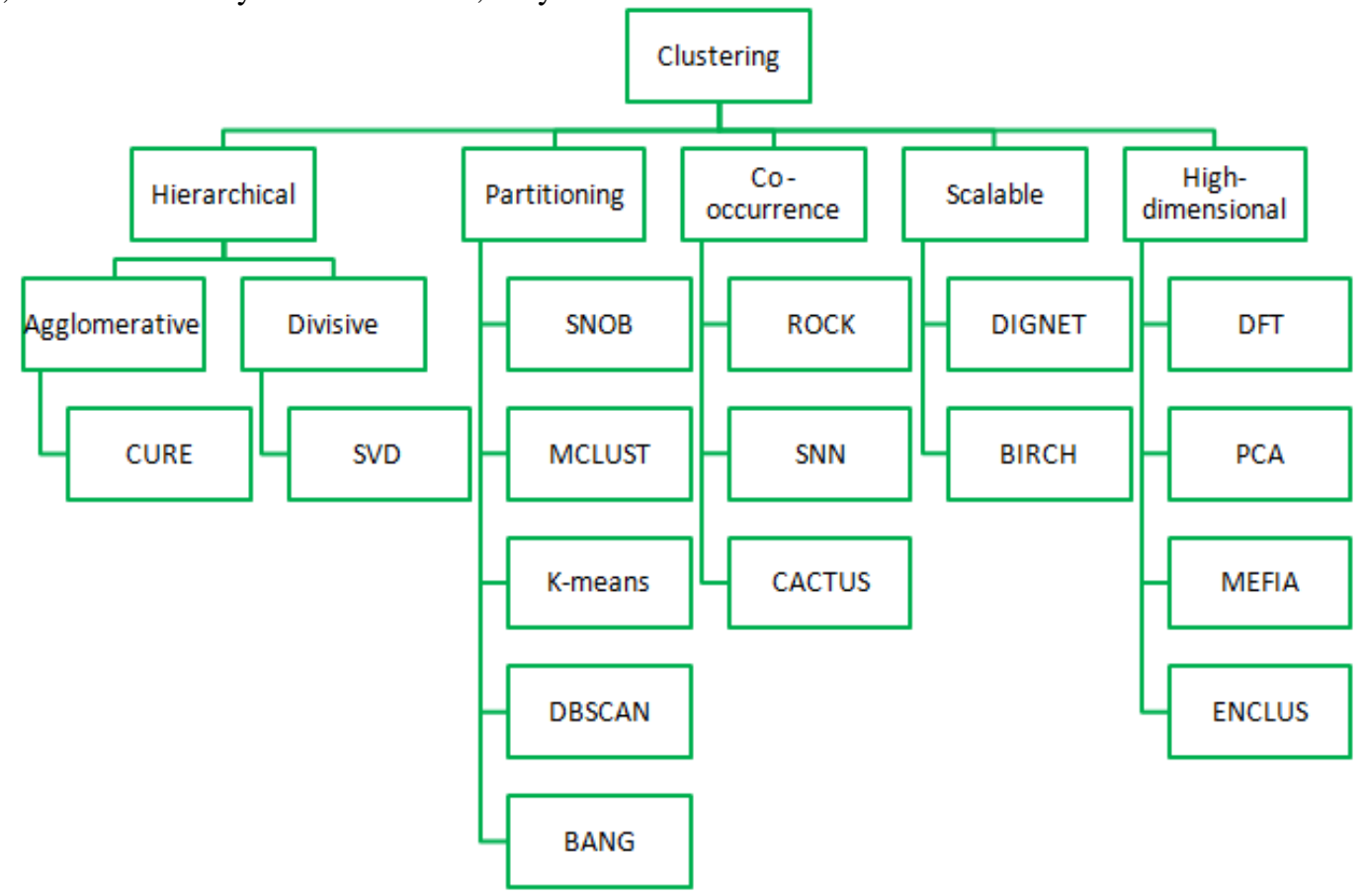

Figure 4. The research structure of Clustering

2.2. Clustering : Grouping calculations partition data into necessary gatherings in order that examples within the same gathering are comparative in some sense and examples in varied gathering are distinctive in an exceedingly similar 
sense[16]. Hierarchal clustering method consolidates info objects into subgroups. Those subgroups converge into larger additionally, abnormal state gatherings et cetera and form a series of importance tree. Numerous leveled grouping ways have 2 arrangements, agglomerative (base up) and divisive (top-down) methodologies. The collective grouping begins with one-point bunches and recursively combines a minimum of 2 of the teams[12]. The divisive grouping conversely may be a top-down technique. The related research incorporates disagreeable person, MCLUST, k-medoids, and k-implies related research. Thickness primarily based apportionment techniques endeavor to search out low-dimensional info that is dense referred to as special knowledge[12]. The related research incorporates DBSCAN (Density based spatial clustering of Applications with Noise). Framework primarily based parceling calculations utilize progressive agglomeration. Collectively amount of making ready and perform house division and subsequently total fitting portions appearance into incorporate BANG. High dimensionality data grouping techniques are intended to modify data with several qualities, counting DFT and MAFIA.

\section{CHALLENGES COMBINED IN THE IOT AND BIG DATA:}

With the quick improvement of IoT, huge data and cloud Computing the foremost principal check is to analyze the expansive volumes of \{data of knowledge and concentrate valuable data or learning for future activities [30]. The key qualities of the knowledge in IoT time may be thought of as massive information; they're as takes once.

- Large volumes of information to peruse and compose: the sum of information can be TB (terabytes), even PB (peta bytes) and $\mathrm{ZB}$ (zeta byte). so we have to investigate quick and viable components.

- Heterogeneous info sources \{and info and knowledge and data kinds to incorporate: in monumental information time the data sources square measure different[29]. For illustration we've to include sensors info cameras, info web-based social networking etc. information measure numerous in arrangement, byte, twofold, string, number, thus forth. we'd like to talk with numerous styles of gadgets and distinctive frameworks and likewise got to concentrate info from web site pages.

- Complex info to separate: The educational is deeply coated up in intensive volumes and also the information isn't clear thus we've to interrupt down the properties and find out the affiliation of varied information[28][29].

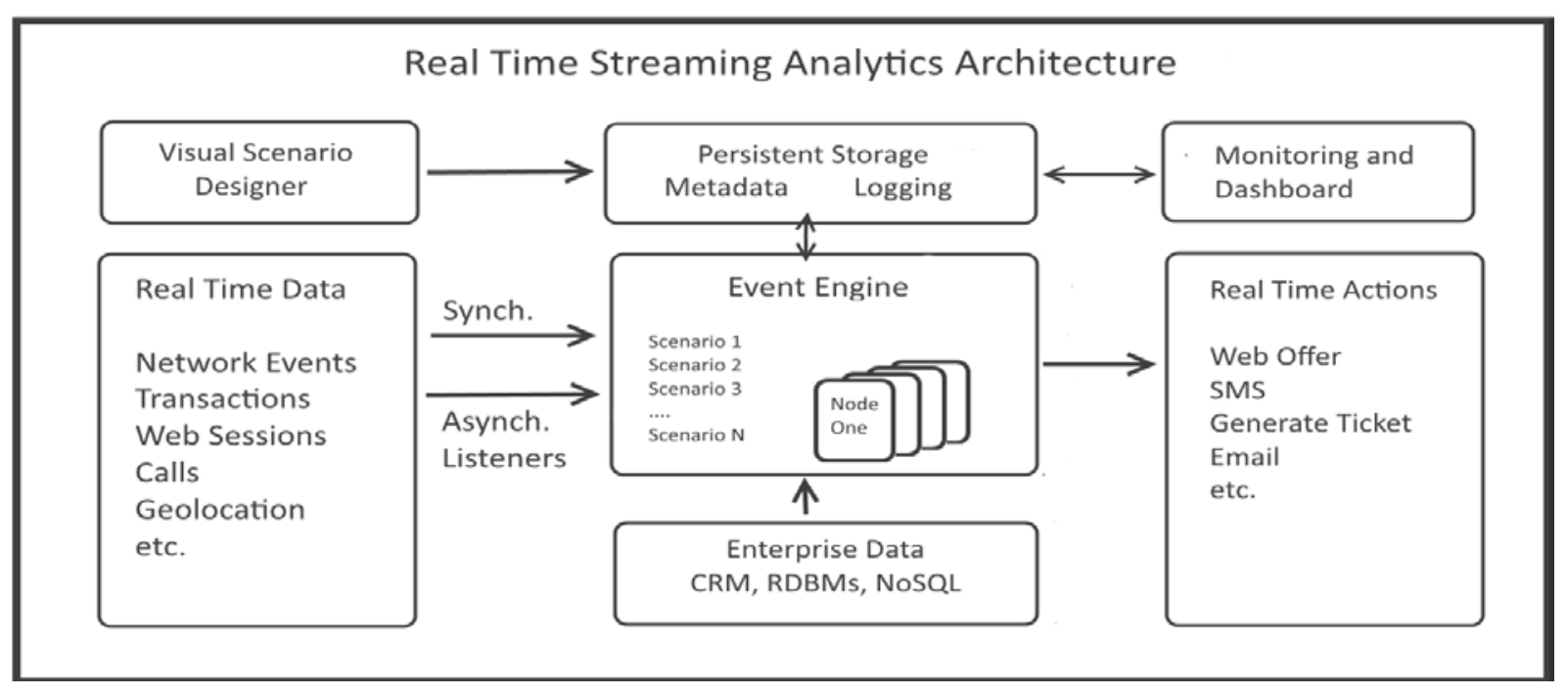

Figure 5. Real Time Streaming Analysis Architecture

\subsection{Challenges:}

Huge number of devices are interconnected they all produce the data continuously from various information sources. IoT also share with Cloud and generating heterogeneous formatted data. Amount of data in massive size so that we want store the data in data node network where managing easily .Whenever we are preferring for data node networks the mining process will goes into analytical process.

- The main test is to urge to extricating substantial scale info from completely different info stockpiling locations. we'd like to manage the assortment, heterogeneity and clamor of the knowledge and it's a major take a look at to find the blame and even tougher to regulate the knowledge. In data mining calculations territory how to amendment customary calculations to very large info condition could be a major test[25].
- Second test is that the means that by that to mine questionable and fragmented data for huge data applications[27]. In data mining framework and security answer for share data between varied applications and frameworks may be a standout amongst the foremost essential difficulties since touchy knowledge as an example keeping cash exchanges and restorative records, got to involve concern.

\subsection{Open Research Issues:}

In big data time there are some open inquire about issues including information checking parallel programming demonstrate and enormous information mining structure.

- There are heaps of inquires about on discovering blunders covered up in information for example 
likewise the information cleaning, sifting, what's more lessening systems are presented.

- Parallel programming model is accustomed to data mining and a number of calculations square measure received to be connected in it[28]. Specialists have extended existing data mining techniques from multiple points of read as well as the proficiency modification of single-source learning revelation techniques outlining an data mining system from a ulti supply purpose of read and therefore the review of dynamic data mining techniques and therefore the investigation of stream data. as an example, parallel affiliation runs mining and parallel k-implies calculation visible of Hadoop stage square measure nice observe[29]. In any case there square measure still a number of calculations that don't seem to be adjusted to parallel stage this demand on applying data mining innovation to large data stage. This is able to be a check for data processing connected specialists and likewise an unbelievable course.

3.3.Big Data Mining System for IoT: The data mining process for vast organizations as Facebook, google, and Twitter etc ., very complicated with traditional approaches . A new set of tools and software are requesting and they will create new infrastructure . Big data mining infrastructure consisting of many new tools and software .

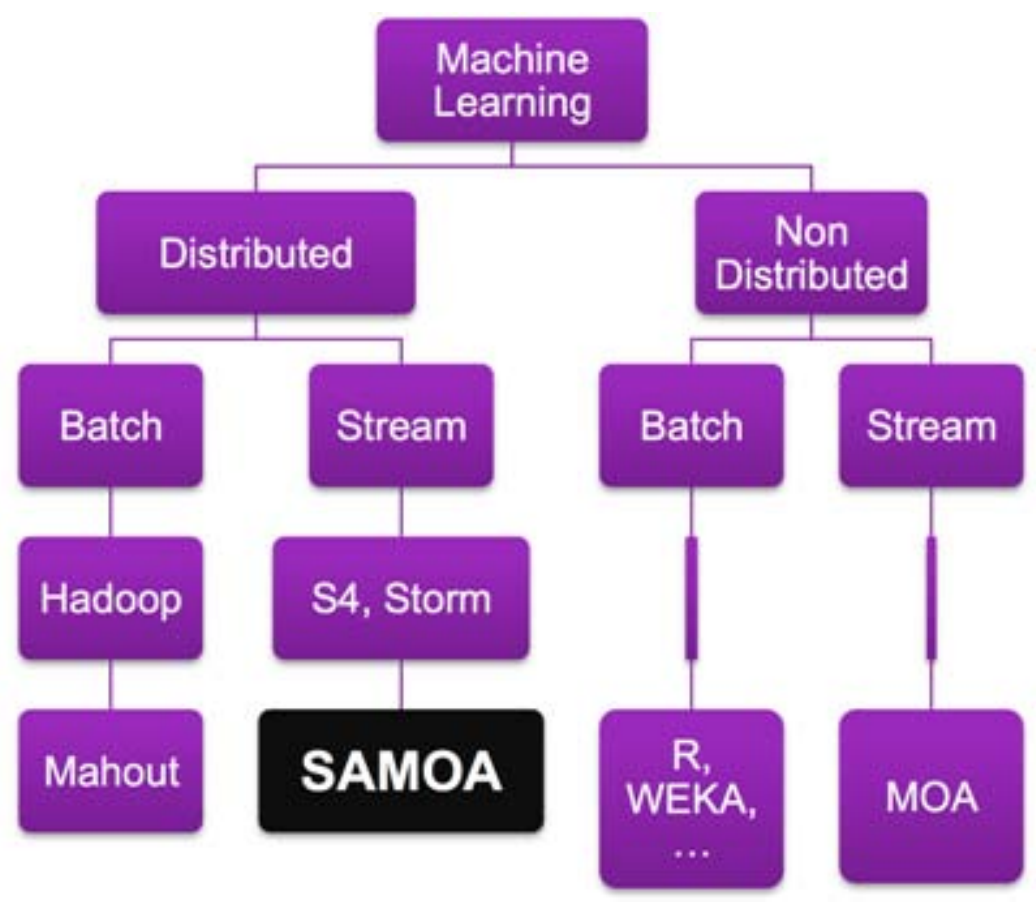

Figure 6. Machine Learning architecture .

- Apache Mahout was enhanced with extensive variety of data mining tools and machine learning calculations.

- MOA enhanced the performance of data mining gradually and also SAMOA enhanced with integrates MOA along with S4 and Strom.

- Hadoop is a integration of tools which can only do analytics, mining and store the data of IoT . IoT becomes a big framework to produce huge data , it is a networks smart nodes. So that we need a special mining technique and mechanisms for mining and analytics. That all tools and software should be satisfy the goals of IoT which is integration of devices and data. The framework design for analytics of IoT data is integration of mining process, storage process and retrieve process . demonstrates a design for the support of social system and distributed computing in IoT. That framework with included tools coordinated the massive value information then perform KDD process that extract, mining and other related functions on data .

3.4. Recommended System Architecture for IoT . we recommend the framework design for IoT and massive data mining framework. in this framework architecture explain with 5 layers. 


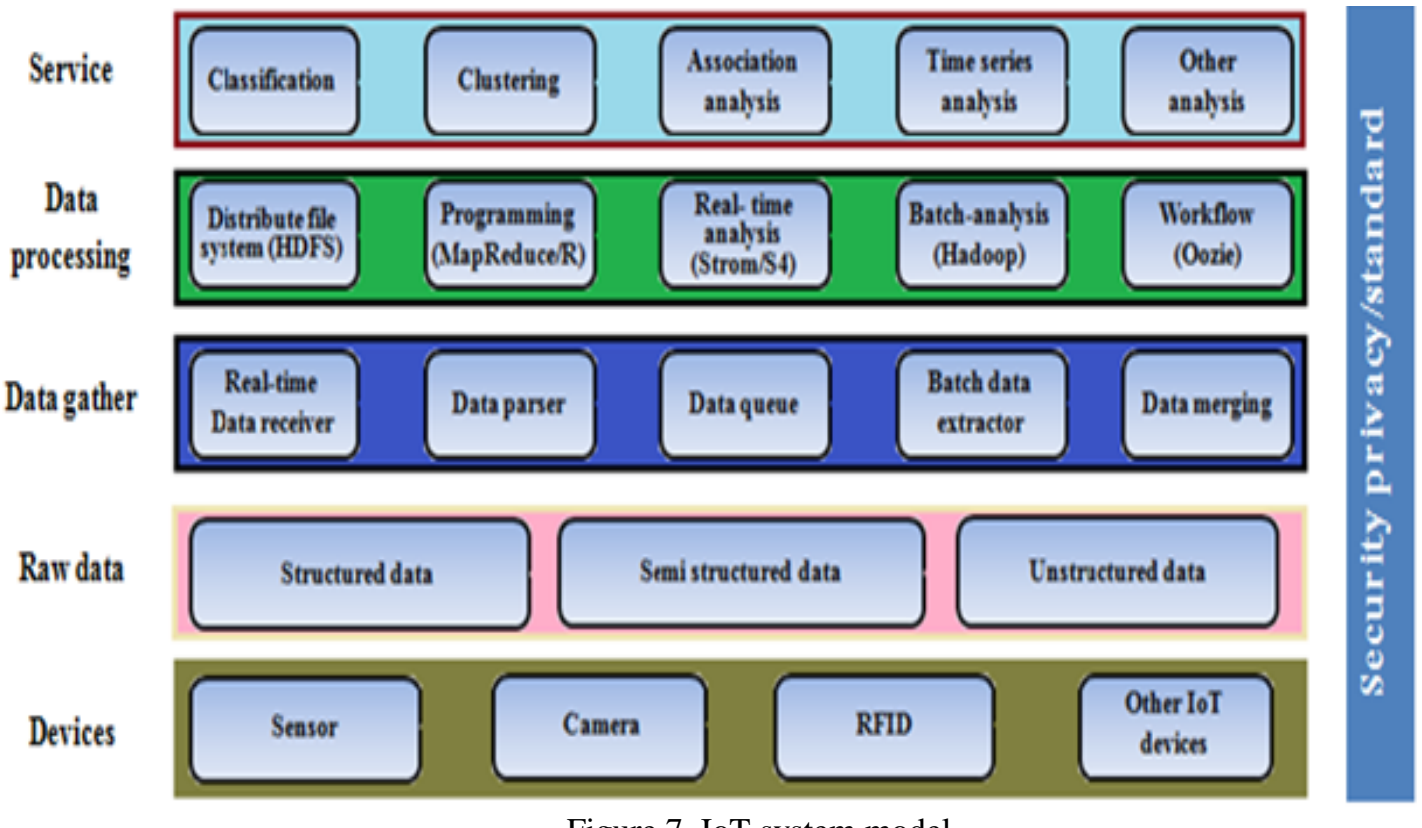

Figure 7. IoT system model .

- devices: It tell about IoT devices like sensors,cameras, RFID, and different smart devices can able to coordinated into IoT framework .

- Raw data: IoT devices produced data in different formats support for store analytics and organized way such as semi structured data, unstructured data , structured data[24][26].

- Data gathering : Generated data collected to create clusters to make possible to store and retrieve. world Journal of Distributed device Networks.

- Data preparing: bunches of open supply arrangements are incorporated, including Hadoop, HDFS, Storm and Oozie.

- $\quad$ Service: information mining capacities will be given as benefit.

- Security/protection/standard: security, protection and normal area unit important to very large info mining framework. Security and security protect the knowledge from unapproved get to and protection disclosure

\section{CONCLUSIONS}

The Internet of Things created new challenges in era of science in technology and their components are intelligent devices, sensors, actuators and self computing devices all they are recognize and investigate by IoT network . All at once to create unfortunate choices each for the things in IoT data mining . Data mining contains techniques of finding patterns to KDD . During this paper we want say about data mining in distinct perspectives information and application in IoT infrastructure . In IoT data mining it can capable to do audit order, bunching, analysis ,retrieve, time arrangement examination and anomaly investigation .This paper tells regarding about learning perspective application for IoT data mining, IoT mining related layers and overview about IoT big data analytics. We tend to examine the new attributes of massive data , difficulties in data filtering and knowledge mining framework. This review of the paper inquire regarding about large data mining in IoT framework

\section{REFERENCES:}

[1] Q. Jing, A. V. Vasilakos, J. Wan, J. Lu, and D. Qiu, "Security of the internet of things: perspectives and challenges,” Wireless Networks, vol. 20, no. 8, pp. 24812501, 2014.

[2] C.-W. Tsai, C.-F. Lai, and A. V. Vasilakos, "Future internet of things: open issues and challenges," Wireless Networks, vol. 20, no. 8, pp. 2201-2217, 2014.

[3] H. Jiawei and M. Kamber, Data Mining: Concepts and Techniques, Morgan Kaufmann, 2011

[4] A. Mukhopadhyay, U. Maulik, S. Bandyopadhyay, and C. A. C. Coello, "A survey of multiobjective evolutionary algorithms for data mining: part I,” IEEE Transactions on Evolutionary Computation, vol. 18, no. 1, pp. 4-19, 2014.

[5] Y. Zhang, M. Chen, S. Mao, L. Hu, and V. Leung, "CAP: crowd activity prediction based on big data analysis," IEEE Network, vol. 28, no. 4, pp. 52-57, 2014.

[6] M. Chen, S. Mao, and Y. Liu, "Big data: a survey," Mobile Networks and Applications, vol. 19, no. 2, pp. 171-209, 2014.

[7] M. Chen, S. Mao, Y. Zhang, and V. Leung, Big Data: Related Technologies, Challenges and Future Prospects, SpringerBriefs in Computer Science, Springer, 2014.

[8] J. Wan, D. Zhang, Y. Sun, K. Lin, C. Zou, and H. Cai, "VCMIA: a novel architecture for integrating vehicular cyber-physical systems and mobile cloud computing," Mobile Networks and Applications, vol. 19, no. 2, pp. 153160, 2014

[9] X. H. Rong, F.Chen, P. Deng, and S. L.Ma, “Alarge-scale device collaboration mechanism," Journal of Computer Research and Development, vol. 48, no. 9, pp. 1589-1596, 2011.

[10] F. Chen, X.-H. Rong, P. Deng, and S.-L.Ma, “A survey of device collaboration technology and system software," Acta Electronica Sinica, vol. 39, no. 2, pp. 440-447, 2011.

[11] L. Zhou, M. Chen, B. Zheng, and J. Cui, "Green multimedia communications over Internet of Things," in Proceedings of the IEEE International Conference on Communications (ICC’12), pp. 1948-1952, Ottawa, Canada, June 2012.

[12] P. Deng, J. W. Zhang, X. H. Rong, and F. Chen, “A model of large-scale Device Collaboration system based on PICalculus for green communication,” Telecommunication Systems, vol. 52, no. 2, pp. 1313-1326, 2013.

[13] P. Deng, J. W. Zhang, X. H. Rong, and F. Chen, "Modeling the large-scale device control system based on PI-Calculus," 
Advanced Science Letters, vol. 4, no. 6-7, pp. 2374-2379, 2011.

[14] J. Zhang, P. Deng, J. Wan, B. Yan, X. Rong, and F. Chen, "A novel multimedia device ability matching technique for ubiquitous computing environments,” EURASIP Journal on Wireless Communications and Networking, vol. 2013, no. 1, article 181, 12 pages, 2013.

[15] G. Kesavaraj and S. Sukumaran, "A study on classification techniques in data mining," in Proceedings of the 4th International Conference on Computing, Communications and Networking Technologies (ICCCNT '13), pp. 1-7, July 2013.

[16] S. Song, Analysis and acceleration of data mining algorithms on high performance reconfigurable computing platforms [Ph.D. thesis], Iowa State University, 2011.

[17] J. R. Quinlan, "Induction of decision trees," Machine Learning, vol. 1, no. 1, pp. 81-106, 1986.

[18] J. R. Quinlan, C4. 5: Programs for Machine Learning, vol. 1, Morgan Kaufmann, 1993.

[19] M. Mehta, R. Agrawal, and J. Rissanen, SLIQ: A Fast Scalable Classifier for Data Mining, Springer, Berlin, Germany, 1996. [20] B. Chandra and P. P. Varghese, "Fuzzy SLIQ decision tree algorithm," IEEE Transactions on Systems, Man, and Cybernetics, Part B: Cybernetics, vol. 38, no. 5, pp. 1294-1301, 2008.

[20] J. Shafer, R.Agrawal, and M.Mehta, "SPRINT: a scalable parallel classifier for data mining," in Proceedings of 22nd International Conference on Very Large Data Bases, pp. 544-555, 1996.

[21] Research on key technologies of data processing in internet of things

To cite this article: Yangqing Zhu and Peiying Liang $2017 \mathrm{~J}$.

Phys.: Conf. Ser. 887012047
[22] Chang, V., 2015. Towards a big data system disaster recovery in a Private cloud. Ad Hoc Networks, 000, pp.1-18.

[23] Sakr, S. \& Gaber, M.M., 2014. Large Scale and big data: Processing and Management Auerbach, ed., Schilling, D.R., 2014. Exaflop Computing Will Save the World ... If We Can Afford It - Industry Tap.

[24] González-Martínez, J. a. et al., 2015. cloud computing and education: A state-of-the-art survey. Computers \& Education, 80, pp.132-151.

[25] D. Matolak: Channel Modeling for Vehicle-to-Vehicle Communications. IEEE Comm.Mag., pp. 76-83, May 2008

[26] V. Subramanian, P. C. Chang, D. Huang, J. B. Lee, S. E. Molesa, D. R. edinger, and S. K.Volkman, "All-printed RFID Tags: Materials, Devices, and Circuit Implications", VLSI Design, 2006.

[27] Chen Qiang1,*,Guang-Quan2, Bai Yu3andLiu Yang2,Researchon Security Issues of the Internet of Things , International Journal of Future Generation Communication and Networking, Vol.6, No.6(2013), pp.1-10

[28] F. Wuhib, R. Stadler, and M. Spreitzer, "Gossip-based resource management for cloud environments," in Proc. CNSM, 2010.

[29] R.S. Barga, J. Ekanayake, W. Lu, Project Daytona: Data Analytics as a Cloud Service, in: A. Kementsietsidis, M. A. V. Salles (Eds.), Proceedings of the International Conference of Data Engineering (ICDE 2012), IEEE Computer Society, 2012, pp. 1317-1320.

[30] B. Franks, Taming The Big Data Tidal Wave: Finding Opportunities in Huge Data Streams with Advanced Analytics, first ed., in: Wiley and SAS Business Series, Wiley, 2012. 